\title{
Problemas de salud mental en alumnos universitarios de primer año de Concepción, Chile
}

\author{
Cristhian Pérez-Villalobos*, Carmen Bonnefoy-Dibarrat, Adherys Cabrera-Flores, Shila Peine-Grandón, Katerina-Macaya \\ Abarca, Marjorie Baqueano-Rodríguez y Jorge Jiménez-Espinoza
}

Universidad San Sebastián (Chile)

\begin{abstract}
Resumen: El presente estudio busca analizar los problemas de salud mental que presentan los alumnos universitarios que ingresan al primer año en una universidad privada de Concepción, Chile. Para esto se encuestó a 554 alumnos ingresados en 2009, evaluando en ellos: sintomatología depresiva a través del Inventario de Depresión de Beck, BDI-II; sintomatología ansiosa mediante el Inventario de Ansiedad de Beck, BAI, y Consumo problemático de alcohol, mediante el Test de identificación de trastornos debidos a consumo de alcohol, AUDIT. Los resultados muestran prevalencias mayores a las documentadas en la literatura, y relaciones de estas variables con género, establecimiento de origen y religión. Lo anterior parece ilustrar el peso específico que el primer año tiene en la vida de los universitarios. Palabras claves: Salud mental; depresión; ansiedad; consumo del alcohol; educación superior.
\end{abstract}

Title: Mental health problems of freshmen in Concepción, Chile Abstract: The aim of this research is to analyze mental health problems in university fressmen in a private institution in Concepcion, Chile. 554 students were surveyed, using Beck Depression Inventory to assess depressive sintomatology; Beck Anxiety Inventory to assess anxious sintomatology and AUDIT to evaluate disorders related to alcohol problematic use. Results show higher prevalences of these problems than showed in previous researches, and also show relations bettween these three variables and gender, type of former school and religion. This results remark the especific relevance of the first year in university to students life.

Keywords: Mental health; depression; anxiety; alcohol comsuption; higher education.

\section{Introducción}

Dentro de las políticas públicas de los países, la salud mental se ha posicionado como una dimensión relevante de la salud, por el impacto que tiene en el funcionamiento cotidiano de los sujetos, en su calidad de vida y por la creciente prevalencia de los problemas en ésta área.

Sólo a nivel lationamericano se ha estimado que las afecciones psiquiátricas explicarían una quinta parte de los años de vida ajustados por discapacidad, lo que contrasta con resultados de diez años antes en que su capacidad explicativa estimada bordeaba el 8\% (Kohn et al., 2005). En México, los trastornos neuropsiquiátricos están en el quinto lugar de las enfermedades asociadas a muerte prematura y días vividos con discapacidad, y en el cuarto puesto entre las enfermedades más incapacitantes (Medina-Mora et al., 2003).

En el caso chileno, un estudio de Vicente, Kohn, Saldivia, Rioseco y Torres en 2005 encontró que un $17.7 \%$ de la población chilena había recurrido a algún tipo de atención de salud mental no especializada, siendo en su mayoría mujeres. En otro estudio, realizado en cuatro provincias del país, el mismo equipo encontró que los trastornos más prevalentes en la población adulta chilena eran, individualmente, la agorafobia, la depresión mayor, la distimia y la dependencia del alcohol. El grupo más frecuente era el del los trastornos ansiosos (trastornos de pánico, agorafobia sin pánico, ansiedad generalizada y estrés postraumático). Al comparar por sexos, se encontró que los trastornos afectivos y ansiosos eran más frecuentes en mujeres y los de abuso de sustancia en los hombres (Vicente, Rioseco, Saldivia, Kohn y Torres, 2002).

Dirección para correspondencia [Correspondence address]: Cristhian Pérez Villalobos. Carlos Oliver 5409, Villa Perales, Talcahuano, Chile. E-mail: cperezvillalobos@me.com

\section{Salud mental en estudiantes universitarios}

El presente estudio busca analizar los problemas de salud mental presentes en estudiantes de pregrado de una universidad privada de la ciudad de Concepción, dado que el ambiente universitario presenta un contexto óptimo para detectar factores de riesgo, trastornos y realizar actividades de prevención, compensación o incluso recuperación (George et al., 1994-1995). Lo anterior es especialmente relevante si se considera que la formación de pregrado tiene lugar en la adolescencia y adultez joven, momento en el que suelen aparecer problemas de salud mental con mayor frecuencia que en etapas anteriores, y en donde aparecen conductas de consumo abusivo, se consolidan patrones desadaptativos y aumenta el riesgo de presentar trastornos alimentarios (Cova et al., 2007). Esto, aunque algunos aspectos internos y externos pueden actuar como factores protectores evitando conductas de riesgo y fortaleciendo la salud mental de los adolescentes, tales como la inteligencia, el apoyo familiar o la religión (Florenzano, 1997)

Además de las características atribuidas directamente a la etapa del desarrollo que vive el universitario, el ambiente académico en sí mismo puede ser nocivo al generar un alto nivel de estrés en los alumnos dadas las altas exigencias intelectuales, sociales y emocionales que conlleva. Perales, Sogi y Morales (2003), refiriéndose específicamente a los alumnos de medicina, sostienen que la vida universitaria se caracteriza por una exigencia académica permanente y en incremento, que demanda continuamente en los estudiantes nuevos esfuerzos de adaptación. Lo anterior, es aplicable también al resto de las carreras de pregrado, donde es necesario enfrentar múltiples estresores, como la sobrecarga académica, dado el número de horas invertido en clases y el tiempo que el estudiante debe destinar de forma independiente para estudiar (Kember, 2004 en Figueiredo-Ferraz, Cardona y Gil-Monte, 2009). 
En este sentido, existen estudios que muestran los alumnos universitarios pueden presentar una serie de trastornos psicológicos al ingresar a la universidad, tales como depresión, obsesión y pérdida de atención, lo que se acentúan en aquellos sujetos que se muestran más nostálgicos (Beck, Taylor y Robbins, 2002).

Ante este escenario, la necesidad de evaluar problemas de salud mental en población universitaria aparece como una actividad esencial para asegurar la calidad de los procesos educativos, toda vez que la salud mental de los alumnos puede influir en su capacidad para lograr aprendizajes de calidad y mantenerse en el sistema, y más allá, si se considera que este puede ser un momento clave para detectar trastornos que pueden afectar la calidad de vida futura del alumnado.

El presente estudio se enmarca en este propósito, como una iniciativa de la Universidad San Sebastián para diagnosticar la salud mental de su estudiantado para diseñar e implementar actividades preventivas y remediales para mejorar su calidad de vida actual o futura. Específicamente, este diagnóstico se concetró en los tres problemas de salud mental más prevalentes en población latinoamericana y chilena, y que han mostrado ser también los más prevalentes en universitarios: depresión, ansiedad y consumo problemático de alcohol (Cova et al., 2007; Balanza, Morales, Guerrero y Conesa, 2008).

\section{Depresión en universitarios}

La depresión es un trastorno afectivo que se caracteriza por un estado de ánimo bajo, anhedónico, poco tolerante, con una mayor probabilidad de presentar sentimientos de culpa y una alteración general de la capacidad de dar y recibir afecto de los demás. Suele asociarse a trastornos del sueño, disminución de la energía y malestares físicos (Riveros, Hernández y Rivera, 2007). En la adolescencia, etapa en la que aún se encuentra la mayoría de los universitarios, se asocia a problemas funcionales y a un mayor riesgo de tener trastornos depresivo en etapas posteriores (Melipillán, Cova, Rincón y Valdivia, 2008).

De acuerdo a Arrivillaga, Cortés, Goicochea y Lozano (2003), su aparición estaría asociada a antecedentes familiares y personales de depresión, ocurrencia de eventos críticos, consumo de alcohol, planeación y/o intento suicida, y también a dificultades académicas.

En un estudio de Vicente et al. (2002), la prevalencia de este trastorno en población adulta chilena fue de $9.0 \%$ durante la vida y $4.6 \%$ durante los últimos seis meses.

A nivel de estudiantes universitarios, en una investigación en Bogotá se encontró que un 25\% de los encuestados presentaba algún nivel de depresión según la escala autoaplicada de Zung y un 30\% según el Inventario de Depresión de Beck (Arrivillaga et al., 2003). En Chile, en tanto, un estudio de Cova et al. (2007) realizado en estudiantes de la Universidad de Concepción, encontró que la prevalencia de trastornos depresivos, utilizando la segunda versión del Inventario de Depresión de Beck, BDI-II, era de un 22\% en mujeres y de un $10.6 \%$ en hombres.

\section{Ansiedad en universitarios}

Otro problema frecuente en la población chilena, y también en la subpoblación universitaria, son los trastornos ansiosos (Cova et al., 2007; Vicente et al, 2002). La ansiedad es una reacción emocional que las personas presentan al tener que adaptarse a eventos que anticipan como aversivos o peligrosos (Celis et al., 2001), y constituye un mecanismo adaptativo natural ante este tipo de situaciones, lo que es adecuado al hacernos suficientemente precavidos $y$, en un nivel moderado, nos ayuda a mantener la concentración y afrontar los retos que tenemos por delante (Riveros et al., 2007). En este sentido, la ansiedad es parte de la vida cotidiana de las personas, y solo constituye un trastorno cuando su duración o intensidad se vuelven incapacitantes. Esto ocurre cuando el sistema de respuesta de la ansiedad se desborda y funciona incorrectamente, lo que hace que la respuesta sea desproporcionada a la situación, en términos de intensidad y/o duración, paraliza al sujeto, lo hace sentir indefenso y deteriora su funcionamiento psicosocial y fisiológico. Es en este punto en el que se le considera un trastorno (Riveros et al., 2007).

La tendencia a la ansiedad tiene efectos que afectan a otros procesos psicológicos de forma negativa, como la vigilia, la atención, la percepción, el razonamiento y la memoria. Además, afecta la vida de las personas, el deteriorar la capacidad de resolución de problemas, las interacciones con otras personas, la calidad de vida y, por consecuencia, la salud en general (Hernández, Coronado, Araújo, y Cerezo, 2008).

La prevalencia de vida de este trastorno en población chilena adulta es de $17.9 \%$ y de un $9.7 \%$ en los últimos seis meses (Vicente et a., 2002) En universitarios se encontró que la prevalencia de trastornos ansiosos era de un $23.8 \%$ en mujeres y de un $10.7 \%$ en hombres, utilizando el Inventario de ansiedad de Beck, BAI, que mide principalmente manifestaciones somáticas de la ansiedad (Cova et al., 2007).

\section{Consumo de alcohol}

Pese a que la mayoría de los estudios de salud mental en universitarios se ha centrado en aspectos emocionales, principalmente depresivos y ansiosos (Cova et al., 2007), un tercer problema de salud mental altamente prevalente en la población, principalmente entre los hombres, es el consumo excesivo de alcohol (Vicente et al. 2002).

En educación superior tampoco es un problema menor, pues el ingreso a la universidad, está asociado al aumento en el consumo de alcohol en los adolescentes, a una ingesta que se caracteriza por detenerse sólo ante la embriaguez, poniendo en riesgo su salud e involucrándose en actividades de alto riesgo (Londoño y Valencia, 2008).

El consumo excesivo de alcohol es un patrón que aumenta la probabilidad que el sujeto enfrente consecuencias 
negativas en su salud física o mental, y/o también para su entorno circundante. En este sentido, la Organización Mundial de la Salud identifica el consumo excesivo sólo con un mayor riesgo asociado sin necesidad de que se haya experimentado aún algún problema (Babor, Higgins, Saunders y Monteiro, 2001).

Este trastorno presenta una elevada comorbilidad psiquiátrica tanto con la ansiedad como la depresión (Balanza et al., 2007). En efecto, el consumo de alcohol puede ser un intento de automedicación del alumno para protegerse de la depresión, o a la inversa, esta puede ser una consecuencia a nivel del sistema nervioso central del consumo de alcohol (Arrivillaga et al, 2003) En este sentido, Arrivillaga et al. (2003) plantean que todos los jóvenes que presentan algún grado de depresión, manifiestan conductas de consumo de alcohol, y que a mayor frecuencia de consumo mayor severidad del estado depresivo.

En cuanto a las causas, un factor de riesgo identificado por Salazar y Arrivillaga (2004) son los antecedentes de los padres, pues en un estudio encontraron que los adolescentes con padres que consumen alcohol tienden a tener prácticas de consumo menos saludable que aquellos cuyos padres no consumen.

Sin embargo, más que la influencia familiar, es necesario reconocer que el uso del alcohol está muy enraizado en culturas como la nuestra. Perales, Sogi y Morales (2003) plantean que en nuestra sociedad cualquier situación fuera de la rutina, tanto triunfos como derrotas, derivan la ingesta de alcohol. En este sentido, Londoño y Valencia (2008) señalan que la presión de grupo se presenta como predictor del consumo de alcohol en los jóvenes, y a pesar de que éstos posean la capacidad de negarse frente esta presión, consumen de igual modo, ya que la cultura les ha mostrado que ésta es una conducta que no genera mayores problemas, no identificándose razones claras para oponerse a la ingesta.

En relación a su prevalencia, en su estudio realizado con estudiantes universitarios del Perú, se encontró una prevalencia de vida de uso que oscilaba entre un $89.8 \%$ y un 95.6\%, dependiendo de la universidad estudiada, mientras que la prevalencia de abuso oscilaba entre el $57.8 \%$ y el $55.7 \%$ (Perales et al., 2003). Estos resultados son similares a los obtenidos por Salazar y Arrivillaga (2004) en universitarios colombianos, donde un $83.8 \%$ respondió haber consumido cigarrillo al menos una vez en la vida.

Específicamente en relación al consumo problemático, en los estudiantes peruanos - evaluado con el CAGE - era de $14.0 \%$ y $12.4 \%$ en cada una de las universidades encuestadas, lo que si bien es coincidente con otros estudios epidemiológicos, no deja de preocupar, dada su asociación a conductas violentas y accidentes (Perales et al., 2003).

\section{Objetivo del estudio}

Los antecedentes anteriores ilustran como la salud mental puede afectar poderosamente el desarrollo y la calidad de vida de las personas, y es, a su vez un indicador de ésta última. De igual modo, se muestra la relevancia de evaluarla en la adolescencia, específicamente en un momento crítico como el ingreso a la universidad en que los alumnos deben comenzar un proceso de adaptación que se extenderá por algunos años.

Debido a lo anterior, el presente artículo se propone como objetivo analizar los problemas de salud mental en alumnos de primer año de una universidad privada de Concepción, para lo cual describirá los niveles y prevalencia de trastorno asociados a las tres problemáticas más frecuentes en la población: depresión, ansiedad y consumo de alcohol. Posteriormente, se evaluará la relación de estos tres problemas con algunas características de los alumnos, tales como sexo, edad y religión.

\section{Método}

La presente investigación, cuantitativa y de alcance correlacional, se realizó a través de un diseño no experimental transversal.

\section{Participantes}

Se encuestó alumnos de pregrado matriculados en 2009 en primer año en programas de pregrado de una universidad privada, quienes fueron elegidos aleatoriamente mediante un muestreo probabilístico estratificado por carrera. Se logró un tamaño muestral de 554 estudiantes, de los cuales se eliminó a 10 por tener más del $5 \%$ de valores perdidos entre sus respuestas. En la muestra resultante, un $58.6 \%$ eran mujeres $(n=319)$ y un $41.3 \% \quad(n=225)$ eran hombres, presentando edades entre los 18 y 34 años $(M=19.96$; D.E. $=2.31)$.

Los alumnos provenían de 30 carreras correspondientes a 33 programas de pregrado diferentes (tres de las carreras se impartían en dos campus de la universidad), cuyas representaciones oscilaron entre 4 alumnos $(0.7 \%)$ en el caso de la carrera de odontología y 28 sujetos $(5.1 \%)$ en el caso de pedagogía en matemáticas, con un promedio de 18.47 participantes por programa.

Un 78.7\% $(n=436)$ de los estudiantes reportó estar cursando su primera carrera, un $18.8 \%(n=104)$ dijo haber estudiado una carrera anterior sin haberla terminado y un $1.3 \%$ $(n=7)$ dijeron haber estudiado y terminado una carrera previa. Otros siete sujetos no respondieron esta pregunta.

En relación a los establecimientos de origen, un 41.6\% $(n=226)$ reportó provenir de establecimientos de enseñanza media de dependencia municipalizada, un 40.4\% $(n=220)$ de establecimientos particulares subvencionados y un $18.0 \%$ $(n=98)$ de establecimientos particulares pagados.

Finalmente, un $57.4 \%$ reportó profesar una religión $(n=318)$, un $41.9 \%(n=232)$ dijo explícitamente no hacerlo y un . $7 \%(n=4)$ no respondió la pregunta. Entre los profesantes, el grupo mayoritario lo constituían los católicos $(n=197$; $35.6 \%$ ), seguido de los que se definían genéricamente como evangélicos $(n=63 ; 11.2 \%)$. 


\section{Instrumentos}

Los sujetos respondieron una batería de cuestionarios de siete cuestionarios, que tributaban a un proyecto de investigación más amplio. En este artículo se presentan sólo los resultados obtenidos de los tres instrumentos que evaluaban problemas de salud mental más el cuestionario sociodemográfico:

- Inventario de Depresión de Beck-II, BDI-II: Diseñado originalmente por Beck, Steer y Brown (1996, citado en Sanz, Perdigón y Vázquez, 2003) y adaptado al español por Sanz, Navarro y Vázquez, (2003, citado en Sanz, Perdigón y Vázquez, 2003), el BDI-II es la versión actualizada de la escala BDI, y se ha convertido en uno de los instrumento más utilizados la práctica clínica y en investigación para evaluar sintomatología depresiva en adultos y jóvenes mayores de 13 años (Cova et al., 2007).

La escala está formada por 21 ítems con cuatro alternativas a las que se asignan puntajes de cero a tres, donde un mayor puntaje indica mayor sintomatología depresiva. Además, el autor propone un puntaje de corte de 19 puntos para identificar trastornos depresivos (Beck et al., 1996),

A diferencia de la primera versión, el BDI-II es más consistente con los criterios diagnósticos sintomáticos de los trastornos depresivos propuestos por el DSM-IV, contando con una versión validada en Chile que ha mostrado una confiabilidad y estructura factorial semejantes a las obtenidas en estudios internacionales (Melipillán, Cova, Rincón y Valdivia, 2008).

En el presente estudio, el instrumento mostró una confiabilidad, según el coeficiente Alfa de Cronbach, de .876 .

- Inventario de Ansiedad de Beck, BAI: Es un inventario de autoinforme de 21 ítemes, que responde a la necesidad de contar con un instrumento que mida el nivel de ansiedad, discriminando entre ésta y la sintomatología depresiva (Steer, Rissmiller y Ranieri, 1993). Según Cova et al. (2007) el puntaje de corte de 16 puntos sugerido por Beck et al. (1993) también resulta adecuado para discriminar entre población con ansiedad normal y patológica en Chile.

Si bien fue diseñado para ser usado fundamentalmente en población clínica, el estudio realizado por Sanz y Navarro (2003) sobre las propiedades psicométricas de la versión española del BAI en universitarios españoles mostró resultados positivos respecto a su consistencia interna (coeficiente alfa de Cronbach de 0.88) y a su capacidad de identificar ansiedad patológica en la población no clínica. En la muestra de la presente investigación el BAI obtuvo un coeficiente Alfa de Cronbach de .899 .

- Test de Identificación de los Trastornos Debidos al Consumo de Alcohol, AUDIT: Elaborado por la Organización Mundial de la Salud (OMS) como un instrumento de screening para detectar consumo excesivo de alcohol, y especialmente para identificar a aquellas personas que podrían beneficiarse con un menor consumo. El instrumento consta de 10 preguntas en las que se evalúa nivel del consumo, dependencia y la presencia de problemas asociados al consumo de alcohol. Para responderlas el participante debe elegir entre cinco alternativas (codificadas desde 0 a 4) que representan niveles variables de ocurrencia de los fenómenos preguntados, aunque siempre en orden de menor a mayor frecuencia. Se considera que un puntaje mayor a 8 puntos indicaría un consumo de riesgo (10 puntos si se quiere mayor precisión, aunque menor sensibilidad), debiendo restarse un punto al puntaje de corte en caso de mujeres y mayores de 65 años (Babor, Higgins-Biddle, Saunders y Monteiro, 2001). El instrumento mostró una confiabilidad de Alfa $=.845 \mathrm{en}$ este estudio.

- Cuestionario sociodemográfico: Este instrumento de preguntas cerradas preguntaba por el sexo y edad de los participantes, además de consultar por antecedentes académicos y la religión que profesaban, en caso de hacerlo.

\section{Análisis de datos}

Para el procesamiento de los datos se realizó un análisis estadístico descriptivo y posteriormente, para el contraste de hipótesis, se utilizaron pruebas estadísticas no paramétricas: U de Mann Whitney, Kruskal Wallis y coeficiente Rho de Spearman Brown, dada la distribución asimétrica de los puntajes de los tres instrumentos utilizados $\mathrm{El}$ análisis se hizo con el apoyo del Paquete estadístico para las ciencias sociales, SPSS, versión 15.0.

\section{Resultados}

Como primer paso se realizó un análisis descriptivo de los datos, que evidenció, en primer lugar, una distribución asimétrica positiva en los tres problemas de salud mental ansiedad, depresión y consumo problemático de alcohol -, presentando estadísticos de asimetría positivos superiores a 1. Esto indica que los sujetos de la muestra mostraban una tendencia a presentar puntajes bajos en estos instrumentos, con unos pocos casos que se desviaron del grupo hacia puntajes altos, Tabla 1.

Tabla 1. Descripción de los problemas de salud mental de los alumnos universitarios

\begin{tabular}{|c|c|c|c|c|c|c|c|}
\hline & Min Máx & $M$ & $M D$ & D.E. & R.I. & Asimetria & Curtosis \\
\hline $\begin{array}{l}\text { BAI } \\
\text { (Ansiedad) }\end{array}$ & .0060 .00 & 14.86 & 13.00 & 9.97 & 11.75 & 1.38 & 2.60 \\
\hline $\begin{array}{l}\text { BDI II } \\
\text { (Depresión) }\end{array}$ & $.00 \quad 50.00$ & 11.53 & 10.00 & 7.71 & 10.00 & 1.32 & 2.67 \\
\hline $\begin{array}{l}\text { AUDIT } \\
\text { (Consumo } \\
\text { problemático } \\
\text { de alcohol) }\end{array}$ & .0032 .00 & 5.08 & 4.00 & 5.50 & 7.00 & 1.51 & 2.67 \\
\hline
\end{tabular}


Para diferenciar los niveles normales de los patológicos en la sintomatología depresiva y ansiosa, Beck (Beck et al., 1996 en Cova et al. 2007) propone 19 y 16 puntos como puntaje de corte para el BDI II y el BAI, respectivamente. De esta forma, las personas con resultados superiores a estos límites presentarían manifestaciones indicativas de trastorno. En el presente estudio se utilizaron estas mismas puntuaciones, ya aplicadas a la población universitaria chilena por Cova et al. (2007), lo que permitió identificar que un $38.8 \%$ ( $n=$ 211) de los estudiantes presentaban manifestaciones indicativas de trastorno ansioso y un $16.5 \%(n=90)$ manifestaciones indicativas de trastornos depresivos.

En el caso del AUDIT se consideraron los puntajes de corte propuesto por Babor et al. (2001) de 7 puntos para mujeres y 8 puntos para hombres, encontrándose que un $28.5 \%$ de los universitarios encuestados presentaban niveles correspondientes a consumo problemático de alcohol.

Como segundo paso, y como parte del análisis bivariado del estudio, se buscó evaluar la relación entre los problemas de salud mental de los universitarios y otras de sus características: sexo, edad, establecimiento en que cursó enseñanza media y religión. Dada la distribución asimétrica de las variables, para evaluar estas relaciones se debió recurrir a análisis estadísticos no paramétricos.

En comienzo se evaluaron las diferencias por sexo en los niveles de ambas sintomatologías y en el consumo de alcohol. En los dos primeros casos, utilizando la prueba no paramétrica U de Mann-Whitney, se identificó diferencias estadísticamente significativas entre ambos sexos, resultando que las mujeres $(M=16.49 ; \quad M d=14.00 ; \quad D . E .=7.69$; $R I=12.00)$ presentan un mayor nivel de sintomatología ansiosa que los hombres $(M=12.55 ; M d=10.00 ; D . E .=7.55$; $R I=11.00), Z=-4.15 ; p<.01$. De la misma manera, las mujeres mostraron un mayor nivel de sintomatología depresiva $(M=16.49 ; M d=11.00 ; D . E .=7.55 ; R I=10.00)$ que éstos $(M=12.55 ; M d=8.00 ; D . E .=9.21 ; R I=8.00), Z=-5.22 ; p<$ .001 .

Los resultados anteriores se vieron confirmados al evaluar la relación entre el género y la presencia de trastorno depresivo y trastorno ansioso, para lo cual las puntuaciones del BDI y el BAI se dicotomizaron a través del puntaje corte establecido por Beck et al. (1996, citado en Cova et al., 2007). De esta forma, utilizando la prueba $\chi^{2}$ de Pearson, los resultados arrojaron una relación estadísticamente significativa entre el género y la presencia de trastorno ansioso, $\square{ }^{2}$ $(1, N=544)=15.832 ; \mathrm{p}<.001$ (Coeficiente Phi $=.171)$, evidenciando que, entre los alumnos de primer año, los hombres $(28.89 \%)$ presentaban en menor proporción este trastorno que las mujeres (45.77\%). En el caso de la depresión, también se encontró una relación estadísticamente significativa entre ambas variables, con una mayor proporción de mujeres con manifestaciones indicativas del trastorno $(19.75 \%)$ que hombres (12.00\%), $\chi^{2}(1, N=544)=5.738 ; \mathrm{p}$ $<.05$ (Coeficiente Phi= .103).

Para analizar la asociación entre sexo y consumo problemático de alcohol, se utilizó nuevamente la prueba U de
Mann-Whitney. De esta forma se encontró nuevamente una diferencia estadísticamente significativa entre ambos sexos, sólo que esta vez fueron los hombres $(M=7.13 ; M d=6.00$; D.E. $=6.59 ; R I=8.50)$ quienes presentaron una sintomatología mayor a las mujeres $(M=3.63 ; M d=2.00 ;$ D.E. $=4.01$; $R I=6.00), Z=-6.40 ; p<.001$. Para complementar el análisis anterior, los puntajes del AUDIT fueron dicotomizados de acuerdo al punto de corte establecido por Babor et al. (2001), lo que permitió reafirmar los resultados previos, resultado también una relación estadísticamente significativa entre el sexo de los alumnos y la presencia de consumo problemático de alcohol, $\chi^{2}(1, N=544)=28.939 ; \mathrm{p}<.001$ (Coeficiente Phi= -.231), con una mayor proporción de estudiantes con consumo problemático entre los hombres $(59.1 \%)$ que entre las mujeres $(19.7 \%)$.

Pese a que la muestra representaba un grupo etario relativamente homogéneo ya que todos alumnos pertenecían a primer año, también se evaluó la relación entre sus edades y los problemas de salud mental que presentaban, no encontrándose relaciones significativas con sintomatología ansiosa, $r h{ }_{(533)}=-.011 ; p=.799$, depresiva, $r h{ }_{(533)}=-.004 ; p$ $=.927$, o con consumo problemático de alcohol rho ${ }_{(533)}=$ $.040 ; p=.352$.

De igual forma, se decidió realizar una comparación en los problemas de salud mental de los alumnos que habían egresado de la enseñanza media desde establecimientos municipalizados, particulares subvencionados y particulares pagados. Esto, pues el tipo de establecimiento en Chile se relaciona con el nivel socioeconómico de las familias, de forma que los establecimientos particulares pagados concentran a los grupos de mayores ingresos y capital cultural y los municipalizados concentran a las personas del polo opuesto (Contreras y Elacqua, 2005). Al hacerlo, utilizando el estadístico de Kruskal-Wallis, se encontró que no había diferencia entre estos tres grupos en la sintomatología depresiva que presentaban, $\chi^{2}(2, N=544)=2.969 ; \mathrm{p}=.227$. Sin embargo, se encontraron diferencias en el caso de la sintomatología ansiosa, $\chi^{2}(2, N=544)=6.380 ; \mathrm{p}<.05$, y en el caso del consumo problemático de alcohol, $\chi^{2}(2, N=544)=9.302$; $\mathrm{p}<.05$. En el primer caso, los alumnos de colegios particulares pagados $(M=12.56 ; M d=11.50 ;$ D.E. $=8.00 ; R I=$ 11.00) presentaban niveles inferiores de sintomatología ansiosa al de los egresados de establecimientos particulares subvencionados $(M=14.55 ; M d=12.50 ; D . E .=9.06 ; R I=$ 10.00) y municipalizados $(M=16.16 ; M d=14.00 ;$ D.E. $=$ 11.33; $R I=14.00)$; en el segundo caso, por el contrario, los alumnos de centros municipalizados $(M=4.49 ; M d=2.00$; D.E. $=5.62 ; R I=7.00)$ presentaron un menor consumo problemático de alcohol que los otros centros particulares, tanto pagados $(M=5.54 ; M d=4.00 ; D . E .=5.60 ; R I=7.00)$ como subvencionados $(M=5.47 ; M d=4.00 ; D . E .=5.30 ; R I=$ 7.00).

En cuanto a la religión, al comparar a quienes reportaron explícitamente profesar una religión y quienes dijeron no hacerlo, se encontró que los primeros presentaban un menor consumo de alcohol $(M=4.15 ; M d=2.00 ;$ D.E. $=4.85$; 
$R I=7.00)$ que los segundos $(M=6.33 ; M d=5.00 ; D . E .=6.07$; $R I=8.00), Z=-4.90 ; p<.001$.

No se encontró diferencias en los niveles de depresión, $Z$ $=-.515 ; p=.607, \mathrm{y}$ ansiedad $Z=-1.160 ; p=.246$.

Por último, al evaluar la relación entre los tres problemas de salud mental evaluados en este estudio, se encontró que estas tres mediciones se encontraban asociadas entre sí, aunque en distinta intensidad. Mientras la sintomatología ansiosa y la sintomatología depresiva mostraban una relación positiva intensa, rho ${ }_{(542)}=.606 ; p<0.001$, el consumo de alcohol presentó sólo relaciones positivas débiles, aunque estadísticamente significativas, con los puntajes del BDI-II, rho ${ }_{(542)}=.118 ; p<.01, \mathrm{y}$ con los del BAI, rho ${ }_{(542)}=0.81 ; p<$ .05 .

Tabla 2. Correlaciones entre los problemas de salud mental de los alumnos universitarios.

\begin{tabular}{lllll}
\hline & & 1 & 2 & 3 \\
\hline $\begin{array}{l}\text { 1. BDI II } \\
\text { (Depresión) }\end{array}$ & Rho & $.876^{\mathrm{a}}$ & & \\
& $p$ & & & \\
& $N$ & 544 & & \\
2. BAI (Ansiedad) & $\mathrm{R} h o$ & $.606\left(^{* *}\right)$ & $.899^{\mathrm{a}}$ & \\
& $p$ & .000 &. & \\
& $N$ & 544 & 544 & \\
3. AUDIT (Consu- & $\mathrm{R} h o$ & $.118\left(^{* *}\right)$ & $.081\left(^{*}\right)$ & $.845^{\mathrm{a}}$ \\
mo problemático de & $p$ & .003 & .029 &. \\
alcohol) & $N$ & 544 & 544 & 544 \\
\hline a. Se indica la confiabilidad obtenida & por el instrumento & según el \\
coeficiente Alfa de Cronbach. & & &
\end{tabular}

\section{Conclusiones}

Una de las primeras finalidades del estudio realizado era dar cuenta de la prevalencia de los problemas de salud mental en las muestras estudiadas, obteniéndose proporciones superiores en los estudiantes universitarios a las que se han registrado en otros estudios, ya sea en población adulta o en el mismo tipo de participantes.

En el caso de la depresión se obtuvo una prevalencia del $16.5 \%$, muy por encima de los niveles observados por Vicente et al. (2002) en adultos, aunque similares a los obtenidos por Cova et al. (2007) en otros alumnos universitarios. Sin embargo, es la prevalencia de ansiedad la que duplica las observaciones realizadas por Cova et al. (2007) que indicaban que un $23.8 \%$ de las mujeres y un $10.7 \%$ de los hombres presentaban manifestaciones indicativas de este diagnóstico: En nuestro estudio, esos porcentajes aumenta a $45.77 \%$ y $28.89 \%$ respectivamente.

¿Cómo se pueden explicar estas diferencias? El estudio de Cova se realizó con estudiantes universitarios de distintos niveles, mientras que la presente investigación se realizó exclusivamente con alumnos de primer año. Lo anterior puede ser un factor relevante, pues el ingreso a la universidad constituye en sí mismo un quiebre biográfico para el alumno, donde aquellos que deben dejar sus casas para empezar sus estudios deben cambiar completamente su entorno físico y social, y aquellos que se mantienen viviendo en casa de sus padres, de todas formas deben rearticular sus redes sociales, pues dejan atrás total o parcialmente el contexto de enseñanza media en donde forjaron algunas de las redes de apoyo más importantes de la adolescencia. En ambos casos, los alumnos de primer año deben hacer frente una transformación social radical a la que deben adaptarse.

Con lo anterior, deben encarar las exigencias académicas propias de la universidad que les demandan horas de estudio, cumplimiento de plazos y, dentro de esto, articular sus actividades con las de sus compañeros, dado que la vida universitaria es en grado importante, grupal.

Estas últimas exigencias, si bien se mantendrán constantes o incluso en incremento durante toda la carrera, entran en una relación recíproca con las capacidades de afrontamiento del alumno. Y son éstas últimas las que, por entrenamiento, podrían mejorar en el tiempo ayudando a que los estudiantes experimenten menores niveles de ansiedad y depresión.

En este sentido, es posible que la salud mental de peor calidad que exhibe el grupo estudiado en relación a otros estudios se deba a particularidades del momento en el que fueron evaluados: primer año. Esto, pese a que en términos puntuales se cuido que la aplicación de instrumentos no se realizara en un momento cercano a pruebas sumativas, lo que podría haber incrementado aún más los síntomas.

En relación al consumo problemático de alcohol, este también se situó por sobre las estimaciones de Perales et al. (2003) en población peruana. Lo cual, si bien es preocupante no aparece como inesperado si se considera la facilitación que se le brinda al consumo de alcohol en Latinoamérica y específicamente en Chile, y la función social que esta tiene como indicador de madurez y movilizador de las actividades de recreación en los adolescentes. Nuevamente, es necesario considerar que se está trabajando con jóvenes, en su mayoría recién egresados de la enseñanza media, que comienzan una etapa en la que la comunidad les brinda mayor autonomía y libertad, y en donde la necesidad de mostrar que realmente han crecido puede ser razón suficiente para mantener o incrementar el consumo de alcohol.

Adicionalmente, los estudiantes mostraron niveles altos de problemas de salud mental que podrían estar coadyuvando el consumo de alcohol. De hecho, si bien la relación de las puntuaciones del AUDIT con el BDI-II y el BAI eran débiles, sí alcanzaron significación estadística, lo que muestra que aunque este sea un factor multicausado deriva al menos en parte de estas dos variables. De esta forma, sumado a un entorno social que permite y promueve la ingesta alcohólica, una mayor sintomatología depresiva y ansiosa podrían derivar en un consumo de alcohol acentuado, utilizándolo como vía de escape o como un regular emocional ante el malestar psicológico. A la inversa, el mayor consumo de alcohol expone a los alumnos a un mayor número de conductas de riesgo y disminuye sus capacidades para realizar un afrontamiento estratégico y efectivo de los problemas que deben 
enfrentar, tanto dentro de la universidad como en el resto de sus vidas, lo que podría incrementar sus síntomas.

Este círculo vicioso puede ser de alto riesgo para los estudiantes, sobre todo sí efectivamente es posible generar mejores estrategias de afrontamiento a la vida universitaria a lo largo del tiempo: Si esto es posible, difícilmente estas estrategias podrán desarrollarse si el alumno se encuentra psicológicamente interferido y subyugado a los efectos del alcohol.

Los resultados también arrojaron diferencias de género en estos tres problemas, similares a los registrados en la literatura. Esto mismo apoya que si bien las mujeres presentan mayores niveles de ansiedad y depresión, al parecer presentan conductas asociadas a los problemas de salud mental que son diferentes al consumo de alcohol, lo que puede estar asociado a que la facilitación social del consumo pese a que se ha abierto recientemente a las mujeres, aún sigue diferenciando ambos géneros.

La edad no presentó relaciones con la salud mental de los alumnos, pero sí lo hizo el tipo de colegios del que egresaron. En este sentido, siguiendo la línea explicativa desarrollada previamente, en relación a las altas exigencias académicas de la universidad y la forma en que demandan del alumno desarrollar estrategias de adaptación, es posible pensar la menor sintomatología ansiosa en los alumnos de establecimientos particulares pagados como una consecuencia lógica de la mejor preparación que estos reciben durante la enseñanza básica y media. En Chile, es de conocimiento público y se confirma año a año con las pruebas estandarizadas, que la calidad de la educación que recibe un estudiante en establecimientos privados es superior a la que recibe el resto de la población (Contreras y Elacqua, 2005). Desde esta perspectiva, si un alumno ha debido enfrentarse a un entorno académico retador, ha sido formado para enfrentarlo con éxito y cuenta con un mayor capital cultural, es posible que enfrente el primer año con menor ansiedad, o generen mejores formas de regulación, que quien no ha tenido estas oportunidades. Si asumimos lo anterior, las inequidades de la educación chilena no sólo afectarían el éxito académico en la educación superior, como ya se ha documentado, sino la calidad de vida y la salud mental que los jóvenes tienen al ingresar - y eventualmente al mantenerse - en este nivel. Y adicionalmente, como una cadena de predestinación fatídica, afectaría las reales posibilidades de los grupos más pobres de sacar provecho de la educación y moverse en la estructura social.

En relación a esto mismo, las diferencias encontradas en el consumo problemático de alcohol aparece como contrain-

\section{Referencias}

Arrivillaga, M., Cortés, C, Goicochea, V. y Lozano, T. (2004). Caracterización de la depresión en jóvenes universitarios. Universitas Psicológica, 3 , $17-25$

Balanza, S., Morales, I., Guerrero, J. y Conesa, A. (2008). Fiabilidad y validez de un cuestionario para medir en estudiantes universitarios la asociación tuitivo y contrario a los prejuicios socialmente difundidos en torno al comportamiento de los grupos más humildes. Esto, pues son los alumnos de colegios particulares quienes presentaron un mayor consumo de alcohol. Si bien esto puede estar mediado por el acceso que se tiene al alcohol, abre la puerta a la necesidad de identificar los factores explicativos de este consumo problemático y cuestionar el peso que deberían tener algunos factores protectores asociados al nivel socioeconómico (véase Florenzano, 1997).

Un último elemento indagado dice relación con la religión, que no mostró relaciones estadísticamente significativas con la sintomatología ansiosa o depresiva, pero sí con el consumo de alcohol. Si bien, al menos dentro de los problemas estudiados parece tener una asociación solamente con la conducta, y no con los afectos y la regulación de los mismos, la relación encontrada reafirma su carácter de factor protector, ya documentado en la literatura.

A partir de los resultados encontrados, surge la necesidad de realizar un estudio longitudinal que permita verificar si las características evaluadas corresponden a una característica propia del primer año o muestra la realidad de los alumnos universitarios en general. Asimismo, considerando las exigencias de la universidad y el efecto en la vida de los alumnos, es necesario describir como van cambiando estas variables a lo largo del tiempo. Lo anterior, es el propósito del proyecto de investigación que se está desarrollando y del que este artículo solamente presenta su primera medición.

Sin embargo, la problemática de la salud mental en los alumnos universitarios debe plantearse más allá. En este sentido, parece necesario que la Universidad se plantee la necesidad de apoyar a sus alumnos, iniciando actividades de promoción e intervención de la salud mental en su estudiantado. Y en el caso de las otras casas de estudio superior, iniciar procesos diagnóstico similares al presente, que les permitan conocer su realidad y tomar las medidas pertinentes.

La labor formativa de una universidad depende de la capacidad de los alumnos para hacerse realmente parte de ella y sacarle provecho. Por otro lado, siguiendo los lineamientos actuales en educación, la Universidad debe plantearse el desarrollo de una persona integral, y en este punto, potenciar adultos sanos debe entenderse como parte de su labor: de una verdadera educación superior.

Agradecimiento.- Este artículo presenta los resultados iniciales del Proyecto DGIE 5068: "La salud mental como factor clave en el proceso educativo: Estudio longitudinal", financiado por la Dirección General de Investigación y Extensión de la Universidad San Sebastián (Chile)

de la ansiedad y depresión con factores académico y psicofamiliares durante el curso 2004-2005. Revista española de salud pública, 82, 189-200.

Beck, A., Steer, R. y Brown, G. (1996). BDI-II. Beck Depression Inventory-second edition. Manual. San Antonio: Psychological Corporation. 
Beck, R., Taylor, C. y Robbins, M. (2002) Missing home: sociotropy and autonomy and their relationship to psychological distress and homesickness in college freshmen. Anxiety, stress, and coping. 16, 155-166.

Babor, T., Higgins, J., Saunders, J. y Monteiro, M. (2001). Audit: Cuestionario de identificación de los trastornos debidos al consume de alcohol: Pautas para su utilización en atención primaria. Ginebra: OMS.

Celis, J., Bustamante, M., Cabrera, D., Cabrera, M., Alarcón, W. y Monge, E. (2001). Ansiedad y estrés académico en estudiantes de medicina humana del primer y sexo año. Anales de la facultad de medicina de la Universidad Nacional Mayor de San Marcos, 62, 25 - 30.

Contreras, D. y Elacqua, G. (2005). El desafío de la calidad y equidad en la educación chilena. En foco, 43, 1 - 48.

Cova, F., Alvial, W., Aro, M., Bonifetti, A., Hernández, M. y Rodríguez, C. (2007). Problemas de salud mental en estudiantes de la universidad de Concepción. Terapia psicológica, 25, 105-112.

Figueiredo-Ferraz, H., Cardona, S. y Gil-Monte, P. (2009). Desgaste psíquico y problemas de salud en estudiantes de psicología. Psicologia em estudio, 14, 349-353.

Florenzano, R. (1997). El adolescente y sus conductas de riesgo. Santiago: Universidad Católica de Chile.

George, M., Siraqyan, X., Mores, R., de la Barra, F., Rodríguez, J., López, C. y Toledo, V. (1994). Adaptación y validación de dos instrumentos de pesquisa de problemas de salud mental en escolares de primero básico. Revista de psicología de la Universidad de Chile, 5, 17-26

Hernández, M., Coronado, O., Araújo, V. y Cerezo, S. (2008). Desempeño académico de universitarios en relación con ansiedad escolar y autoevaluación. Acta colombiana de psicología, 11,13-23.

Kohn, R., Levav, I., Caldas, J.M., Vicente, B., Andrade, L., CaraveoAnduaga, J., Saxena, J. y Saraceno, B. (2005). Los trastornos mentales en América Latina y el Caribe: asunto prioritario para la salud pública. Revista Panamericana de Salud Publica, 18, 229-40.

Londoño, C. y Valencia, C. (2008). Asertividad, resistencia a la presión de grupo y consumo de alcohol en universitarios. Acta colombiana de psicologia, $11,155-162$
Melipillán, R., Cova, F., Rincón, P. y Valdivia, M. (2008). Propiedades psicométricas del Inventario de depresión de Beck en adolescentes chilenos. Terapia psicológica, 26, 59-69.

Medina-Mora, M., Borges, G., Lara, C., Benjet, C., Blanco, J., Fleiz, C., Villatoro, J., Rojas, E., Zambrano, R., Casanova, L. y Aguilar-Gaxiola, S. (2003). Prevalencia de trastornos mentales y uso de servicios: Resultados de la encuesta nacional de epidemiología psiquiátrica en México. Revista de Salud Mental, 26, 1-16.

Perales, A., Sogi, C. y Morales, R (2003). Estudio comparativo de salud mental en estudiantes de medicina de dos universidades estatales peruanas. Anales de la Facultad de Medicina de la Universidad Nacional Mayor de San Marcos, 64, 239-246.

Riveros, M., Hernández, H. y Rivera, J. (2007). Niveles de depresión y ansiedad en estudiantes universitarios de Lima metropolitana. Revista de investigación en psicología, 10, 91-102.

Salazar, I. C. y Arrivillaga, M. (2004). El consumo de alcohol, tabaco y otras drogas como parte del estilo de vida de los jóvenes universitarios. Revista Colombiana de Psicología, 13, 74-89

Sanz, J., Perdigón, A. y Vázquez, C. (2003). Adaptación española del Inventario para la Depresión de Beck-II (BDI-II): Propiedades psicométricas en población general. Clínica y salud, 14, 249-280.

Sanz, J. y Navarro, M. (2003). Propiedades psicométricas de una versión española del Inventario de Ansiedad de Beck (BAI) en estudiantes universitarios. Ansiedad y Estrés, 9, 59-84.

Steer, R., Rissmiller, D. y Ranieri, W. (1993). Structure of the computerassisted Beck Anxiety Inventory with psychiatric inpatients. Journal of personality assessment, 60, 532-542.

Vicente, B., Rioseco, P., Saldivia, S., Kohn, R. y Torres, S. (2002). Estudio chileno de prevalencia de patología psiquiátrica. Revista médica de Chile, 130 (5), 527-536.

Vicente, B., Kohn, R., Saldivia, S., Rioseco, P. y Torres, S. (2005). Patrones de uso de servicios entre adultos con problemas de salud mental, en Chile. Revista Panamericana de Salud Publica, 18, 263-270.

(Artículo recibido: 3-5-2010, revisado: 3-4-2012, aceptado: 8-4-2012) 